\title{
Phase Noise Suppression Algorithm Based on Modified LLR Metric in SC-FDMA System
}

\author{
Zijie Xu and Guangliang Ren \\ State Key Laboratory of Integrated Service Networks, Xidian University, No. 2 South Taibai Road, Xi'an 710071, China \\ Correspondence should be addressed to Guangliang Ren; glren@mail.xidian.edu.cn
}

Received 26 January 2017; Accepted 4 April 2017; Published 9 May 2017

Academic Editor: Jit S. Mandeep

Copyright ( 2017 Zijie Xu and Guangliang Ren. This is an open access article distributed under the Creative Commons Attribution License, which permits unrestricted use, distribution, and reproduction in any medium, provided the original work is properly cited.

\begin{abstract}
In single-carrier frequency-division multiple-access (SC-FDMA) receivers in the long-term evolution uplink, phase noise is one of the major radio-frequency front-end impairments that degrade system performance. To address this issue, we propose a new loglikelihood ratio (LLR) computation algorithm. A signal model with residual phase noise is considered in the algorithm. Based on this model, we derive a closed-form expression of the likelihood function of the received symbol and calculate more accurate LLR information. Thus the accuracy of the decoder is increased and the performance of the SC-FDMA system is improved. Simulation results show that our proposed algorithm achieves superior bit error rate (BER) performance compared with the existing LLR calculation algorithms in high-order quadrature amplitude modulations (QAM).
\end{abstract}

\section{Introduction}

To achieve higher spectral efficiencies, the current trend in the long-term evolution (LTE) uplink is to employ high-order modulation schemes, such as 256-ary quadrature amplitude modulation (256-QAM) or 1024-QAM [1]. However, the single-carrier frequency-division multiple-access (SCFDMA) system with high-order modulations is very vulnerable to phase noise (PN), which will break the orthogonality among subcarriers and distort the received signals [2]. Therefore the bit error rate (BER) performance of the SC-FDMA system is seriously degraded. To cope with this impairment, many algorithms have been proposed to suppress PN [36]. A block-type pilot-based intercarrier interference (ICI) suppression algorithm was proposed in [3]. The interference components were directly calculated and the ICI matrix was reconstructed by exploiting block-type pilot. However, the algorithm is impractical since the PN changing rapidly in every SC-FDMA symbol. In [4], a PN suppressing algorithm based on the equalizer was discussed, but this algorithm needs to transmit some symbols twice which will cause high overhead. In [5], a PN estimation algorithm and a way to mitigate the PN effect were proposed. However, due to the effect of the additive white Gaussian noise (AWGN), the estimation of the PN becomes inaccurate which degrades the performance of the algorithm. In [6,7], iterative receivers were introduced to suppress the PN. For such iterative receivers, log-likelihood ratio (LLR) calculation at the demodulator is crucial for the decoder to work properly. A pilot-tone assisted LLR algorithm based on a modified Bessel function was derived in $[8,9]$, where only common phase error (CPE) is considered in the signal model. Another approach [10] was to employ linear transform (LT) on the phase noise term and derive an approximate expression of the likelihood function. By using the likelihood function, a modified LLR metric was obtained. The performance improvement of the decoder is limited due to the inaccurate LLR metric.

In this paper, we focus on the received signal equalized by the pilot in the SC-FDMA receiver. After carrier-phase recovery, the received signal is disturbed by the residual PN and AWGN. Based on this received signal model, we derive an accurate closed-form formula of the likelihood function and propose a modified LLR calculation algorithm. Benefitting from the modified LLR, the decoder works properly and the BER performance of the system is improved. The paper is organized as follows. The phase noise model and the signal model are described in Section 2. The proposed LLR calculation algorithm is derived in Section 3. Simulation 


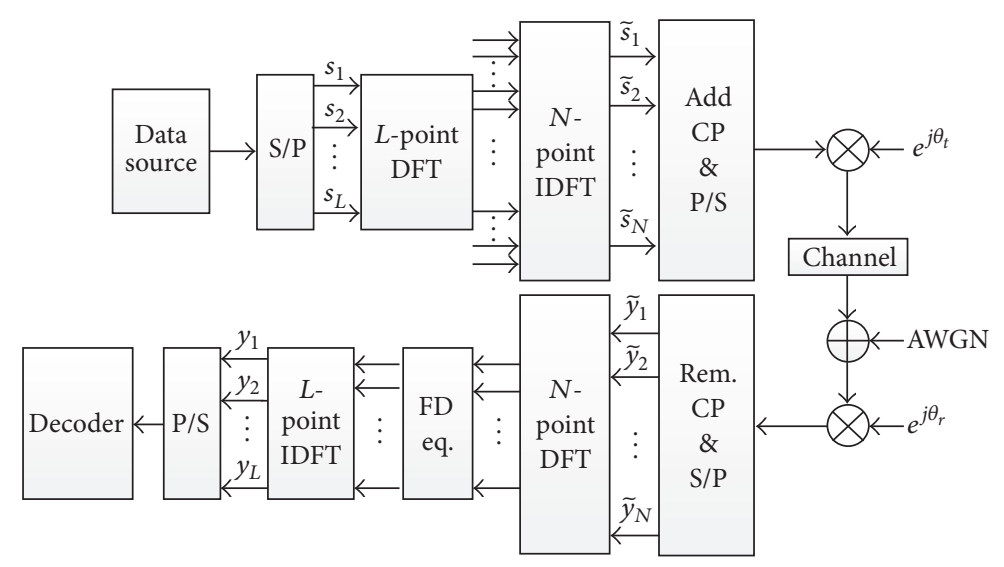

FIGURE 1: Block diagram of the discrete baseband SC-FDMA transceiver.

results are discussed in Section 4 and conclusions are drawn in Section 5.

\section{System Model}

2.1. Phase Noise Model. PN arises from imperfections in the local oscillator (LO) and results in random fluctuations in the phase of LO's output sinusoidal signal. PN is usually modeled in two ways: one is Wiener model generated by the freerunning oscillator [11]. The Wiener-type $\mathrm{PN}$ is nonstationary and its variance increases with time; the other one is phase locked loop (PLL) model which is a stationary zero-mean Gaussian random process [12]. In this paper, we model the residual $\mathrm{PN}$ as a zero-mean Gaussian random process with variance $\sigma_{\theta}^{2}$; that is, $\theta(n) \sim \mathbb{N}\left(0, \sigma_{\theta}^{2}\right)$.

2.2. Signal Model. Figure 1 shows the block diagram of a discrete baseband SC-FDMA transceiver. Assume that there are $N$ subcarriers in the SC-FDMA system, where each user occupies $L(L<N)$ of them. For simplicity and without loss of generality, here we focus on a single user scenario. An $L \times 1$ data vector $\mathbf{s}$ is generated at the transmitter, and $\mathbf{F}_{L}$ denotes an $L \times L$ discrete Fourier transform (DFT) matrix with the element in the $m$ th row and the $n$th column being given by

$$
\left(\mathbf{F}_{L}\right)_{m n}=\left(\frac{1}{\sqrt{L}}\right) e^{-j 2 \pi(m-1)(n-1) / L} .
$$

Similarly, $\mathbf{F}_{N}^{\mathrm{H}}$ denotes an $N \times N$ inverse DFT (IDFT) matrix where $(\cdot)^{\mathrm{H}}$ denotes the matrix Hermitian operation. The subcarrier mapping of the user is defined by the $N \times L$ matrix $\mathbf{T}$ whose elements are zeros, except for a single " 1 " in each column. The indexes of the rows containing these " 1 "s correspond to the locations of the assigned subcarriers for the user. Then the $N \times 1$ SC-FDMA time-domain (TD) transmitted symbol vector $\widetilde{\mathbf{s}}$ is given by

$$
\widetilde{\mathbf{s}}=\mathbf{F}_{N}^{\mathrm{H}} \mathbf{T F}_{L} \mathbf{s}
$$

and the $N \times 1$ TD received symbol vector $\widetilde{\mathbf{y}}$ is given by

$$
\widetilde{\mathbf{y}}=\mathbf{P}_{r} \mathbf{H} \mathbf{P}_{t} \widetilde{\mathbf{s}}+\widetilde{\mathbf{z}}=\mathbf{P}_{r} \mathbf{H} \mathbf{P}_{t} \mathbf{F}_{N}^{\mathrm{H}} \mathbf{T} \mathbf{F}_{L} \mathbf{s}+\widetilde{\mathbf{z}},
$$

where $\mathbf{P}_{t} \triangleq \operatorname{diag}\left(e^{j \theta_{t}(1)}, e^{j \theta_{t}(2)}, \ldots, e^{j \theta_{t}(N)}\right)$ and $\mathbf{P}_{r} \triangleq$ $\operatorname{diag}\left(e^{j \theta_{r}(1)}, e^{j \theta_{r}(2)}, \ldots, e^{j \theta_{r}(N)}\right)$ denote diagonal PN matrices at the transmitter and receiver, respectively. $\theta_{t}(n)$ and $\theta_{r}(n)$ are the PN samples generated by the transmitter and receiver, respectively, at the $n$th sample of the SC-FDMA symbol. $\mathbf{H}$ is the $N \times N$ circulant channel matrix whose first column contains the zero-padded channel impulse response (CIR) between the user and the base station. $\widetilde{\mathbf{z}}$ is the $N \times 1$ complex AWGN vector. After equalization, the $L \times 1$ recovered symbol vector $\mathbf{y}$ is given by

$$
\mathbf{y}=\mathbf{F}_{L}^{\mathrm{H}} \mathbf{E} \mathbf{T}^{\mathrm{H}} \mathbf{F}_{N} \widetilde{\mathbf{y}}
$$

where $\mathbf{E}$ is the frequency domain (FD) equalization matrix. When we apply FD minimum mean square error (MMSE) equalization, we have $\mathbf{E} \triangleq \operatorname{diag}\left(\left\{\overline{\mathbf{H}}^{\mathrm{H}}(n, n) /\left(|\overline{\mathbf{H}}(n, n)|^{2}+\right.\right.\right.$ $\left.\left.\rho^{-1}\right)\right\}_{n=1}^{N}$ ), where $\overline{\mathbf{H}}$ is the equivalent diagonal FD channel matrix obtained from the pilot symbols and $\rho$ is the signal-tonoise ratio (SNR) of the transmitted signals. Note that $\overline{\mathbf{H}}$ is the result of the combination of the channel and the phase noise at the pilot symbols; we perform PN initial compensation in the FD equalization process. Substituting (3) back into (4), we have

$$
\mathbf{y}=\mathbf{F}_{L}^{\mathrm{H}} \mathbf{E} \mathbf{T}^{\mathrm{H}} \mathbf{F}_{N} \mathbf{P}_{r} \mathbf{H} \mathbf{P}_{t} \mathbf{F}_{N}^{\mathrm{H}} \mathbf{T} \mathbf{F}_{L} \mathbf{s}+\mathbf{F}_{L}^{\mathrm{H}} \mathbf{E} \mathbf{T}^{\mathrm{H}} \mathbf{F}_{N} \widetilde{\mathbf{z}} .
$$

From [6], the recovered symbol vector $\mathbf{y}$ is approximated as

$$
\mathbf{y}=\mathbf{P s}+\mathbf{z},
$$

where $\mathbf{P} \triangleq \operatorname{diag}\left(e^{j \theta(1)}, e^{j \theta(2)}, \ldots, e^{j \theta(L)}\right)$ is the residual $\mathrm{PN}$ matrix, $\theta(n)$ is the residual PN sample (see also in Section 2.1), and $\mathbf{z}=[z(1), z(2), \ldots, z(L)]^{\mathrm{T}}$ is the complex AWGN vector with mean zero variance $\sigma^{2}$; that is, $z(n) \sim \mathbb{C N}\left(0, \sigma^{2}\right)$. Note that residual $\mathrm{PN} \theta(n)$ is independent of $z(n)$. For the sake of simplicity of the following derivation, we write (6) in elemental form and omit the time label as follows:

$$
y=e^{j \theta} s+z
$$




\section{LLR Calculation Algorithm}

3.1. Conventional LLR Calculation Algorithm. Conventional LLR algorithm considers a signal model where only AWGN $z$ is presented [13]:

$$
y=s+z
$$

The LLR of a particular received bit $b$ is defined as

$$
L=\ln \frac{\operatorname{Pr}(b=1 \mid y)}{\operatorname{Pr}(b=0 \mid y)},
$$

where $\operatorname{Pr}(b=1 \mid y)$ and $\operatorname{Pr}(b=0 \mid y)$ denote the probabilities that, given the received symbol $y$, the received bit $b$ should be decoded as " 1 " and the probability that bit $b$ should be decoded as " 0 ", respectively. Assuming that " 1 " and " 0 " bit values occur at equal probabilities, the conventional LLR (CLLR) can be written as

$$
\begin{aligned}
L_{C} & =\ln \frac{\operatorname{Pr}(y \mid b=1)}{\operatorname{Pr}(y \mid b=0)}=\ln \frac{\sum_{s \in S^{1}} p(y \mid s)}{\sum_{s \in S^{0}} p(y \mid s)} \\
& =\ln \frac{\sum_{s \in S^{1}}\left(1 / \pi \sigma^{2}\right) \exp \left(-|y-s|^{2} / \sigma^{2}\right)}{\sum_{s \in S^{0}}\left(1 / \pi \sigma^{2}\right) \exp \left(-|y-s|^{2} / \sigma^{2}\right)},
\end{aligned}
$$

where $S^{1}$ and $S^{0}$ denote subsets of the constellation symbols in which the decoded bit $b$ equals " 1 " and " 0 ", respectively. In order to reduce the computational complexity, Max-LogMAP algorithm [14] is used in (10) and (10) becomes

$$
L_{C}=-\frac{1}{\sigma^{2}}\left(\max _{s \in S^{1}}|y-s|^{2}-\max _{s \in S^{0}}|y-s|^{2}\right) .
$$

From (11) we can see that, for signal model described in (8), the LLR can be obtained by calculating the Euclidean distances between the received symbol $y$ and the nearest constellation symbol in each of subsets $S^{1}$ and $S^{0}$. When it comes to a received signal model with residual $\mathrm{PN}$, as shown in (7), the C-LLR calculation algorithm in (11) will no longer be applicable. Therefore, we derive a modified LLR calculation algorithm in the presence of residual PN.

3.2. Proposed LLR Calculation Algorithm. We focus on the received signal model as described in (7). Conditioned on the residual $\mathrm{PN} \theta$ and $s$, the probability density function (PDF) of received symbol $y$ is

$$
\begin{aligned}
& p(y \mid \theta, s)=\frac{1}{\pi \sigma^{2}} \exp \left\{-\frac{1}{\sigma^{2}}\left|y-e^{j \theta} s\right|^{2}\right\}=\frac{1}{\pi \sigma^{2}} \\
& \cdot \exp \left\{-\frac{1}{\sigma^{2}}\left(|y|^{2}+|s|^{2}\right)\right\} \\
& \cdot \exp \left\{\frac{2}{\sigma^{2}}\left|e^{-j \theta} s^{*} y\right| \cos (\angle y-\angle s-\theta)\right\}=\frac{1}{\pi \sigma^{2}} \\
& \cdot \exp \left\{-\frac{1}{\sigma^{2}}\left(|y|^{2}+|s|^{2}\right)\right\} \\
& \cdot \exp \left\{\frac{2}{\sigma^{2}}|y||s| \cos \left(\varphi_{0}-\theta\right)\right\}
\end{aligned}
$$

where $\varphi_{0} \triangleq \angle y-\angle s$ is the angular difference between the received symbol and the constellation symbol being evaluated. The likelihood function is evaluated by integrating (12) over residual PN $\theta$ :

$$
\begin{aligned}
p(y \mid s) & =\int_{-\infty}^{+\infty} p(y, \theta \mid s) d \theta \\
& =\int_{-\infty}^{+\infty} p(y \mid \theta, s) p(\theta \mid s) d \theta .
\end{aligned}
$$

Because the residual $\mathrm{PN} \theta$ is independent of constellation symbol $s$, that is, $p(\theta \mid s)=p(\theta)$, we can rewrite (13) as

$$
p(y \mid s)=\int_{-\infty}^{+\infty} p(y \mid \theta, s) p(\theta) d \theta
$$

where $p(\theta)=\left(1 / \sqrt{2 \pi} \sigma_{\theta}\right) \exp \left\{-\theta^{2} / 2 \sigma_{\theta}^{2}\right\}$ is the PDF of the residual PN. Substituting (12) into (14), we have

$$
p(y \mid s)=\frac{1}{\pi \sigma^{2} \sqrt{2 \pi} \sigma_{\theta}} \exp \left\{-\frac{1}{\sigma^{2}}\left(|y|^{2}+|s|^{2}\right)\right\} \cdot I
$$

where

$$
I \triangleq \int_{-\infty}^{+\infty} \exp \left\{\frac{2}{\sigma^{2}}|y||s| \cos \left(\varphi_{0}-\theta\right)-\frac{\theta^{2}}{2 \sigma_{\theta}^{2}}\right\} d \theta .
$$

To obtain a closed-form solution of the integral $I$, we introduce the Taylor mean value theorem. The corresponding first-order Taylor expansion for $\cos \left(\varphi_{0}-\theta\right)$ in integral $I$ at $\theta=0$ is given by

$$
\begin{aligned}
\cos \left(\varphi_{0}-\theta\right)= & \cos \varphi_{0}+\sin \varphi_{0} \cdot \theta-0.5 \\
& \cdot \cos \left(\varphi_{0}-\xi\right) \cdot \theta^{2}
\end{aligned}
$$

where $R(\theta) \triangleq-0.5 \cdot \cos \left(\varphi_{0}-\xi\right) \cdot \theta^{2}$ is the Lagrange remainder, and the value of $\xi$ ranges from 0 to $\theta$. Considering $\varphi_{0}-\xi$ approximates to 0 in high SNR scenarios, we can rewrite (17) as

$$
\cos \left(\varphi_{0}-\theta\right)=\cos \varphi_{0}+\sin \varphi_{0} \cdot \theta-0.5 \theta^{2}
$$

Substituting (16) and (18) into (15), we have

$$
\begin{aligned}
& p(y \mid s) \\
& =\frac{1}{\sqrt{2} \pi \sigma \sigma_{\theta}} \cdot \frac{1}{\sqrt{|y||s|+\sigma^{2} / 2 \sigma_{\theta}^{2}}} \\
& \quad \times \exp \left\{\frac{1}{\sigma^{2}}\left[\frac{\left(|y||s| \sin \varphi_{0}\right)^{2}}{|y||s|+\frac{\sigma^{2}}{2 \sigma_{\theta}^{2}}}-|y-s|^{2}\right]\right\} .
\end{aligned}
$$




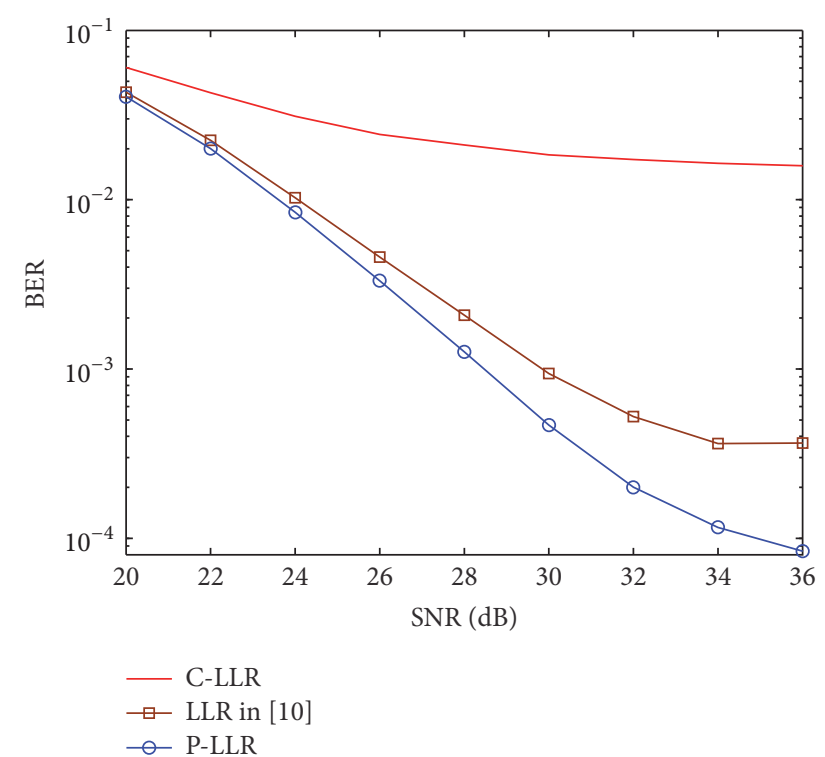

FIGURE 2: BER performance of various LLR algorithms for 256QAM.

Similar to (10) and (11), we use Max-Log-MAP algorithm and obtain the proposed LLR (P-LLR) as

$$
\begin{aligned}
L_{P}= & -\frac{1}{\sigma^{2}} \\
& \left(\begin{array}{l}
\max _{s \in S^{1}}\left(\frac{\left(|y||s| \sin \varphi_{0}\right)^{2}}{|y||s|+\frac{\sigma^{2}}{2 \sigma_{\theta}^{2}}-|y-s|^{2}}\right) \\
-\max _{s \in S^{0}}\left(\frac{\left(|y||s| \sin \varphi_{0}\right)^{2}}{|y||s|+\frac{\sigma^{2}}{2 \sigma_{\theta}^{2}}}-|y-s|^{2}\right)
\end{array}\right)
\end{aligned}
$$

\section{Simulation Results}

Numerical results for the proposed LLR (P-LLR) algorithm are presented in this section. Conventional LLR (C-LLR) and LLR algorithm in [10] are also experimented for comparison. We model SC-FDMA systems with $N=512, L=96$, and the sampling rate is $7.68 \mathrm{MHz}$, as specified in the LTE uplink standard [15] for $5 \mathrm{MHz}$ bandwidth. The data bits are channel coded with the convolutional code of code rate $1 / 2$. The 256-QAM and 1024-QAM modulations are considered. The standard deviation of the residual $\mathrm{PN}$ is set to $\sigma_{\theta}=7^{\circ}$.

Figures 2 and 3 depict the system's BER versus SNR performance for 256-QAM and 1024-QAM with $\sigma_{\theta}=7^{\circ}$, respectively. The BER of various LLR algorithms decreases as SNR grows. It can be seen from the two figures that the BER performance of our P-LLR algorithm is superior to the other two algorithms for both cases. The BER of P-LLR algorithm is reduced to $10^{-3}$ at $\mathrm{SNR}=28 \mathrm{~dB}(256-\mathrm{QAM})$ and $\mathrm{SNR}=$ $36 \mathrm{~dB}$ (1024-QAM). The P-LLR algorithm has about $1.5 \mathrm{~dB}$

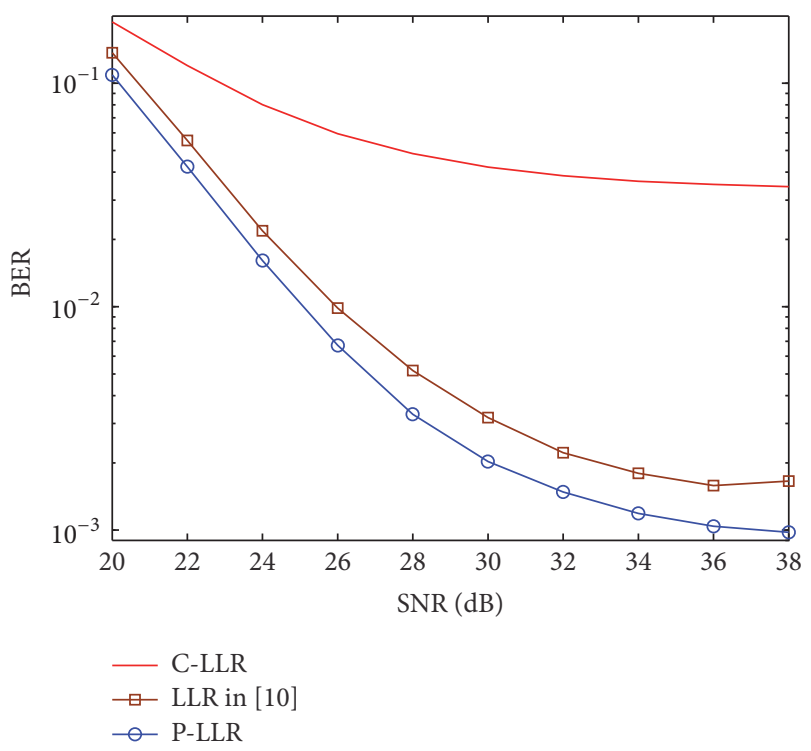

FIGURE 3: BER performance of various LLR algorithms for 1024QAM.

and $3 \mathrm{~dB}$ gain over algorithm in [10] at $\mathrm{BER}=10^{-3}(256-$ $\mathrm{QAM})$ and $\mathrm{BER}=2 \times 10^{-3}$ (1024-QAM), respectively. The LLR algorithm in [10] has a BER error floor of $4 \times 10^{-4}(256-$ QAM) at SNRs above $34 \mathrm{~dB}$ and $1.5 \times 10^{-3}$ (1024-QAM) at SNRs above $36 \mathrm{~dB}$. The C-LLR algorithm is much worse than our P-LLR algorithm and has a BER error floor of $1.6 \times 10^{-2}$ (256-QAM) at SNRs above $36 \mathrm{~dB}$ and $2.5 \times 10^{-2}$ (1024-QAM) at SNRs above $38 \mathrm{~dB}$. The superiority of the proposed P-LLR algorithm in BER performance over C-LLR is due to the fact that C-LLR only considers the AWGN, while P-LLR takes care of both aspects of the AWGN and the residual PN. The performance gain of the P-LLR algorithm compared with LLR algorithm in [10] comes from the application of accurate likelihood function in (19). By applying more accurate LLR, the decoding performance of the decoder is improved and thus the BER of the system is decreased.

\section{Conclusion}

We propose a modified LLR calculation algorithm for the received signal model with residual PN in LTE uplink SCFDMA system whose performance is easily impaired by the PN. By using Taylor mean value theorem, the closed-form formula of the likelihood function is derived. Based on the likelihood function, a modified LLR calculation algorithm is obtained. Compared with existing LLR algorithms, our proposed LLR algorithm achieves superior BER performance. The proposed algorithm can be used in high-order modulation system which meets the increasing demand for ultra-high bit rate in $5 \mathrm{G}$ communication systems.

\section{Conflicts of Interest}

The authors declare that there are no conflicts of interest regarding the publication of this paper. 


\section{Acknowledgments}

This work was supported by the National Natural Science Foundation of China under Grant 91538105 and the National Basic Research Program of China under Grant 2014 CB340206.

\section{References}

[1] P. Banelli, S. Buzzi, G. Colavolpe, A. Modenini, F. Rusek, and A. Ugolini, "Modulation formats and waveforms for $5 \mathrm{G}$ networks: who will be the heir of OFDM?: an overview of alternative modulation schemes for improved spectral efficiency," IEEE Signal Processing Magazine, vol. 31, no. 6, pp. 80-93, 2014.

[2] G. Sridharan and T. J. Lim, "Performance analysis of SC-FDMA in the presence of receiver phase noise," IEEE Transactions on Communications, vol. 60, no. 12, pp. 3876-3885, 2012.

[3] X. Zhang and H.-G. Ryu, "Joint estimation and suppression of phase noise and carrier frequency offset in multipleinput multiple-output single carrier frequency division multiple access with single-carrier space frequency block coding," IET Communications, vol. 4, no. 16, pp. 1998-2007, 2010.

[4] B. R. Sang, K. Jangsu, R. Heung-Gyoon, and L. Yingshan, "PNS algorithm for the SC-FDMA communication system with phase noise," in Proceedings of the 2009 IFIP International Conference on Wireless and Optical Communications Networks (WOCN '09), pp. 1-5, Cairo, Egypt, April 2009.

[5] V. Syrjälä, "Modelling and practical iterative mitigation of phase noise in SC-FDMA," in Proceedings of the 2012 IEEE 23rd International Symposium on Personal, Indoor and Mobile Radio Communications (PIMRC '12), pp. 2395-2400, Australia, September 2012.

[6] A. Gomaa and N. Al-Dhahir, "Phase noise in asynchronous SCFDMA systems: performance analysis and data-aided compensation," IEEE Transactions on Vehicular Technology, vol. 63, no. 6, pp. 2642-2652, 2014.

[7] S. Suyama, H. Suzuki, K. Fukawa, and J. Izumi, "Iterative receiver employing phase noise compensation and channel estimation for millimeter-wave OFDM systems," IEEE Journal on Selected Areas in Communications, vol. 27, no. 8, pp. 13581366, 2009.

[8] S. Cao, P.-Y. Kam, and C. Yu, "Pilot-aided log-likelihood ratio for LDPC coded M-QAM CO-OFDM system," in Proceedings of the Conference on Optical Fiber Communication, Technical Digest Series, San Francisco, Calif, USA, March 2014.

[9] S. Cao, P.-Y. Kam, C. Yu, and X. Cheng, "Pilot-tone assisted log-likelihood ratio for LDPC coded CO-OFDM system," IEEE Photonics Technology Letters, vol. 26, no. 15, pp. 1577-1580, 2014.

[10] T. Koike-Akino, D. S. Millar, K. Kojima, and K. Parsons, "Phase noise robust LLR calculation with linear/bilinear transform for LDPC-coded coherent communications," in Proceedings of the Conference on Lasers and Electro-Optics Europe, pp. 1-2, San Jose, Calif, USA, June 2015.

[11] L. Tomba, "On the effect of wiener phase noise in OFDM systems," IEEE Transactions on Communications, vol. 46, no. 5, pp. 580-583, 1998.

[12] Z. Chen and F. F. Dai, "Effects of LO phase and amplitude imbalances and phase noise on M-QAM transceiver performance," IEEE Transactions on Industrial Electronics, vol. 57, no. 5, pp. 1505-1517, 2010.
[13] X. Wang and H. V. Poor, "Iterative (Turbo) soft interference cancellation and decoding for coded CDMA," IEEE Transactions on Communications, vol. 47, no. 7, pp. 1046-1061, 1999.

[14] A. J. Viterbi, "An intuitive justification and a simplified implementation of the MAP decoder for convolutional codes," IEEE Journal on Selected Areas in Communications, vol. 16, no. 2, pp. 260-264, 1998.

[15] I. T. S. Sesia and M. Baker, LTE-The UMTS Long Term Evolution: From Theory to Practice, Wiley, Hoboken, NJ, USA, 2009. 


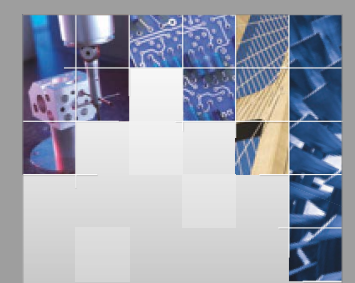

\section{Enfincering}
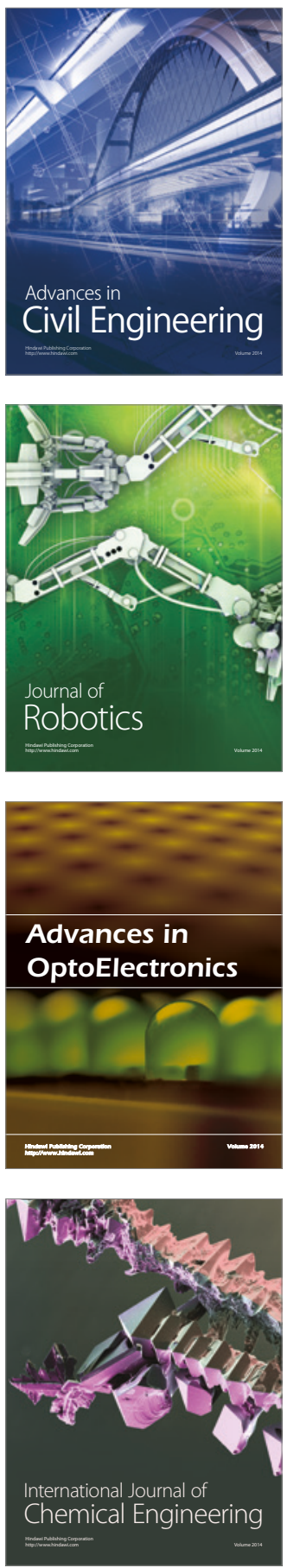

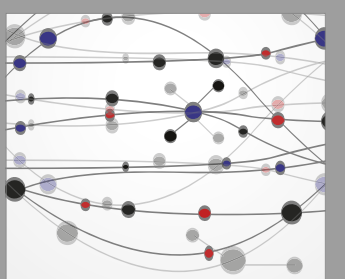

The Scientific World Journal

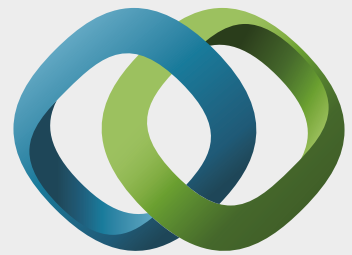

\section{Hindawi}

Submit your manuscripts at

https://www.hindawi.com
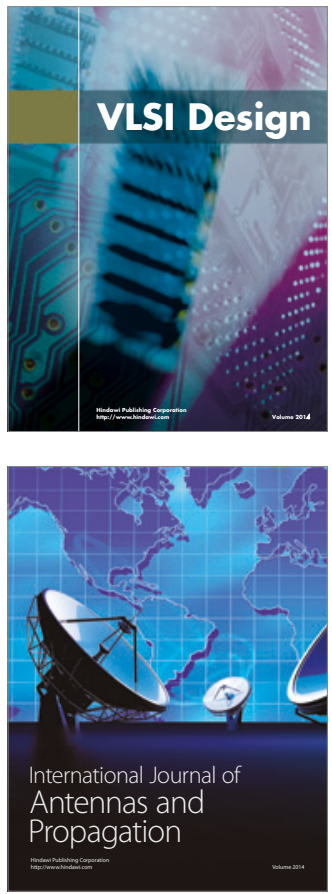

\section{Rotating}

Machinery
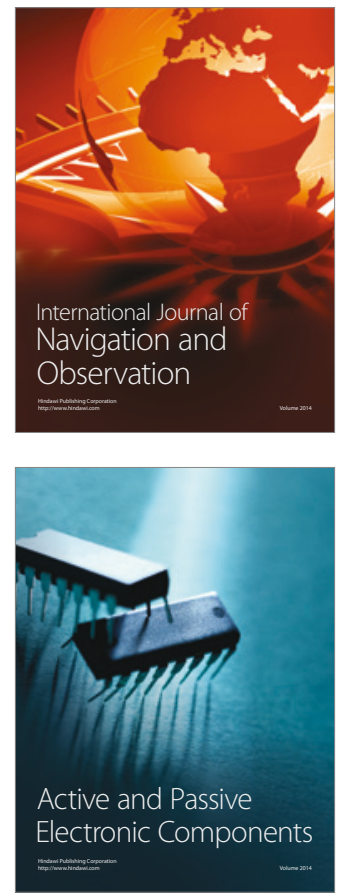
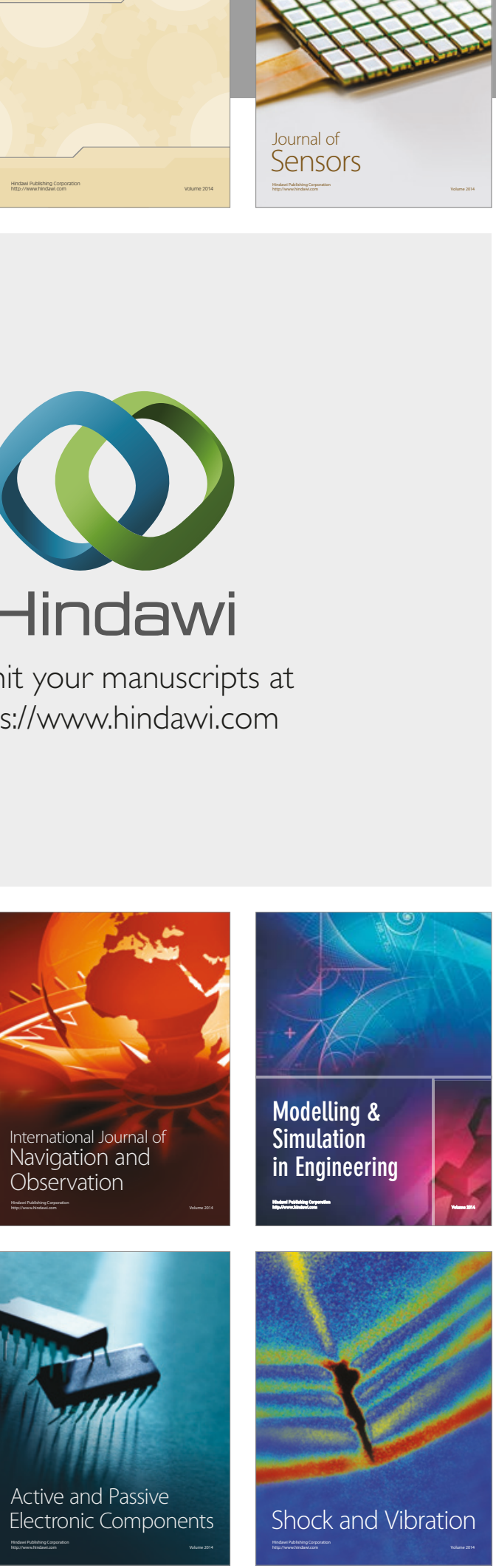
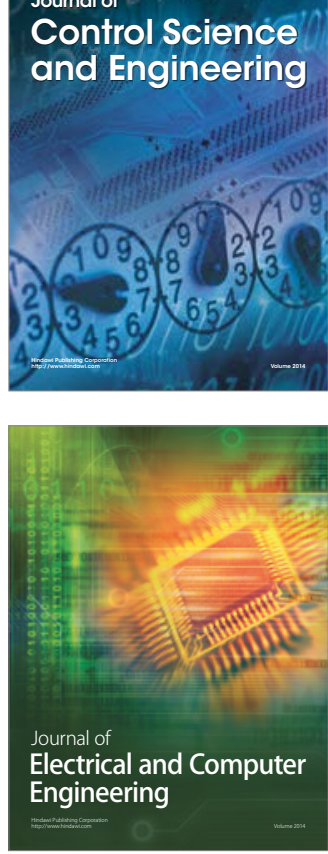

Distributed

Journal of

Control Science

and Engineering
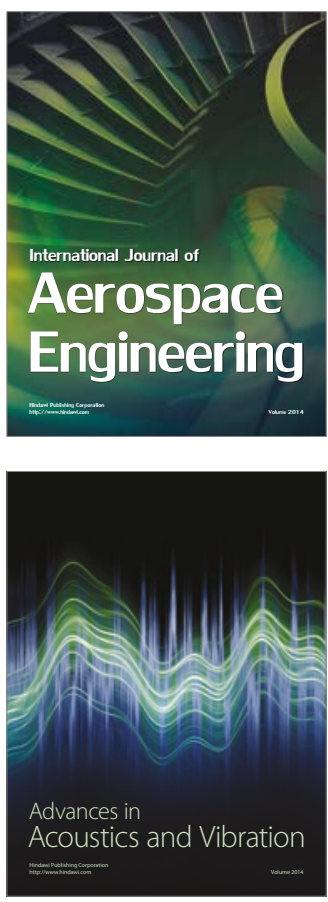

Sensor Networks 